OPEN

SUBJECT AREAS:

METALS AND ALLOYS

MECHANICAL PROPERTIES

APPLIED PHYSICS

TECHNIQUES AND

INSTRUMENTATION

Received

24 June 2013

Accepted

3 December 2013

Published

19 December 2013

Correspondence and requests for materials should be addressed to

Q.B.Y.

lqingboyu1970@

163.com) or X.H.L.

(liuxh@mail.neu.edu.

$\mathrm{cn})$

\section{Extreme Extensibility of Copper Foil under Compound Forming Conditions}

\author{
Qingbo Yu ${ }^{1,2}$, Xianghua Liu' \& Delin Tang'
}

\author{
${ }^{1}$ Research Institute of Science and Technology, Northeastern University, Shenyang 1 10819, China, ${ }^{2}$ Mechanical and Electronic \\ Engineering College, Xuzhou Institute of Technology, Xuzhou 221008, China.
}

A copper foil with an extreme extensibility up to $43,684 \%$ was obtained without any intermediate annealing by means of asynchronous rolling with high tension. It was found that under the combination of compression, shearing and tension, the copper foil represents a wonderful phenomenon. As the reduction increases, the specimen hardness increases up to a peak value $138 \mathrm{HV0.05}$ when the foil thickness rolled to around $100 \mu \mathrm{m}$, and then it decreases down to $78 \mathrm{HV} 0.05$ when the foil thickness rolled to the final size $19 \mu \mathrm{m}$. It tells us that the strain-softening effect occurs when the foil thickness is rolled down to a threshold level. The experimental results bring us some fresh ideas different with the traditional understanding on the strain-hardening mechanism of metals, which provides an experimental basis to establish the forming mechanism of the thin foil.

$\mathrm{t}$ is well known that strain hardening by cold deformation can increase the strength and hardness of metals ${ }^{1-3}$. Is this law still valid when the specimen thickness is thin enough to the level of micrometer by a large cold deformation? It becomes recently an interesting question to material scholars ${ }^{4,5}$. An electrodeposited nanocrystalline $\mathrm{Cu}$ with grain size of $30 \mathrm{~nm}$ was rolled at room temperature by $\mathrm{L}$. Lu et al. $^{6}$, and an extreme extensibility (elongation $>5,100 \%$ ) was obtained. They proposed that the extreme extensibility of the nanocrystalline $\mathrm{Cu}$ originates from a deformation mechanism dominated by grain boundary activities rather than lattice dislocation. This research achievement has attracted attention in materials research ${ }^{7-9}$. Compared to the nanocrystalline $\mathrm{Cu}$ in reference ${ }^{6}$, whether there still is the extreme extensibility for a common coarse-grained $\mathrm{Cu}$ ? The answer was not found at any report until now. Therefore a series of experiment on the special forming, microstructures and properties tests were carried out in order to make it clear. On the other hand, it is of significant to discover the extreme extensibility in the industrial pure $\mathrm{Cu}$, because it provides a potential application in optimization of the technology to produce copper foil to meet the requirements of modern industry, such as micromanufacturing, 3C products, etc. Meanwhile, it also inspires us to search the deep reasons of the wonderful phenomena near the micro size, enabling it to serve the scientific development and human civilization.

\section{Results}

Extreme extensibility. The pure $\mathrm{Cu}$ with the initial thickness $8.3 \mathrm{~mm}$ was rolled to a thin foil with thickness $19 \mu \mathrm{m}$ without any intermediate annealing. At this case the extensibility of the specimen arrives to high level of $43,684 \%$, as shown in Figure 1. The extreme extensibility is related with the compound forming conditions, by a special design rolling mill as shown in Figure 2, especially the shearing function which makes more slip systems activate, participate in slip and cross $\operatorname{slip}^{10}$, and lead to the deformation easier than in common rolling. This is one of the reasons why the microns foil was obtained.

In order to distinguish it from the elongation $\delta$ in uniaxial tension, here the Extensibility in Thickness Ratio(ETR)written as $\eta$, was used to represent the formability of the thin foil:

$$
\eta=\frac{H}{h} \times 100 \%
$$

where the $H$ and $h$ are the foil thickness before and after forming respectively. According to the condition of constant volume, ETR $\eta$ could be express as:

$$
\eta=\frac{b l}{B L}=\frac{b}{B}\left(\frac{l-L}{L}+1\right) \times 100 \%=\frac{b}{B}(\delta+1) \times 100 \% \approx(\delta+1) \times 100 \%
$$




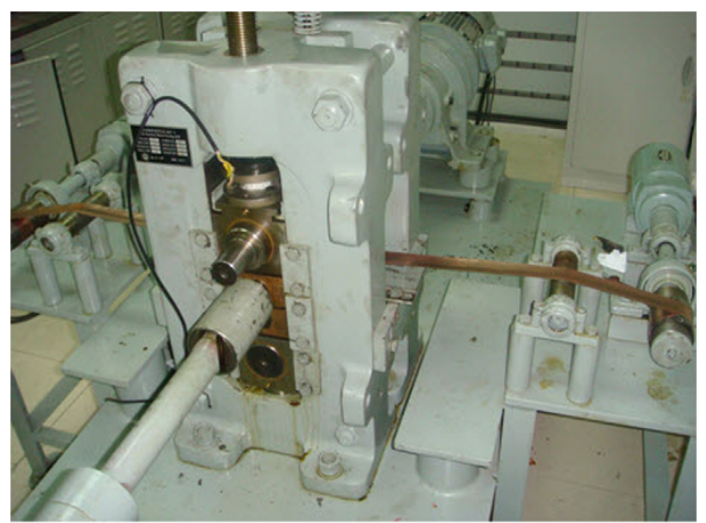

Figure 1 The industrial pure copper specimens before and after rolling. Using asynchronous rolling at room temperature without intermediate annealing, the extensibility up to $43,684 \%$.

where the $B$ and $L$ are the foil width and length before forming, $b$ and $l$ are the width and length after forming respectively. Because the spread during the foil rolling is very little in many cases, it could take $b \approx B$. The ETR $\eta$ of the specimen got in our experiment is as high as $43,684 \%$ (be equivalent to $\delta \approx 43,584 \%$ by Eq. 2 ), with good surface, no cracks on the edge, excellent strip flatness and regular external appearance. We were interested in this data because it is a new record comparing with $5,100 \%$ by reference ${ }^{6}$ in the year 2000 for nanocrystalline copper.

Microstructure and microhardness. In order to understand the reason, the microstructures of the specimens were observed, and it was found that the grains of initial copper before rolling were equiaxed and the average grain size was about $220 \mu \mathrm{m}$, as shown in Figure 3(a). With increasing the ETR $\eta$, the grains were elongated along the rolling direction and squashed in the thickness direction. Meanwhile, the grain shape became lamellar structure and the surface area ratio of the grain was quickly increased. When the ETR $\eta$ was up to $43,684 \%$, the lamellar grains could still be retained, as illustrated in Figure 3(b). Figure 4 shows the grain size in the thickness direction at different ETR $\eta$. It can be seen that the grain lamellae spacing decreases with the increase of ETR $\eta$, but they were far from to the nanometer scale. It indicates that the extreme extensibility in our experiment does not come from the formation of nanocrystalline. What is the reason that makes the specimen obtain so high extensibility? Microhardness measurements showed that the microhardness of the initial copper specimen before forming was 78 HV0.05, as shown in Figure 5. The strain-hardening was remarkable

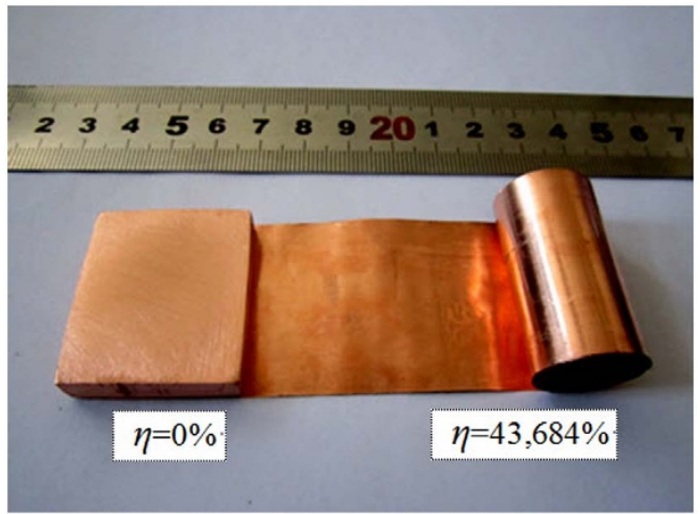

Figure $2 \mid 3$ M-mill, by which the combination function of compression, shearing and tension occurs simultaneously during asynchronous rolling.
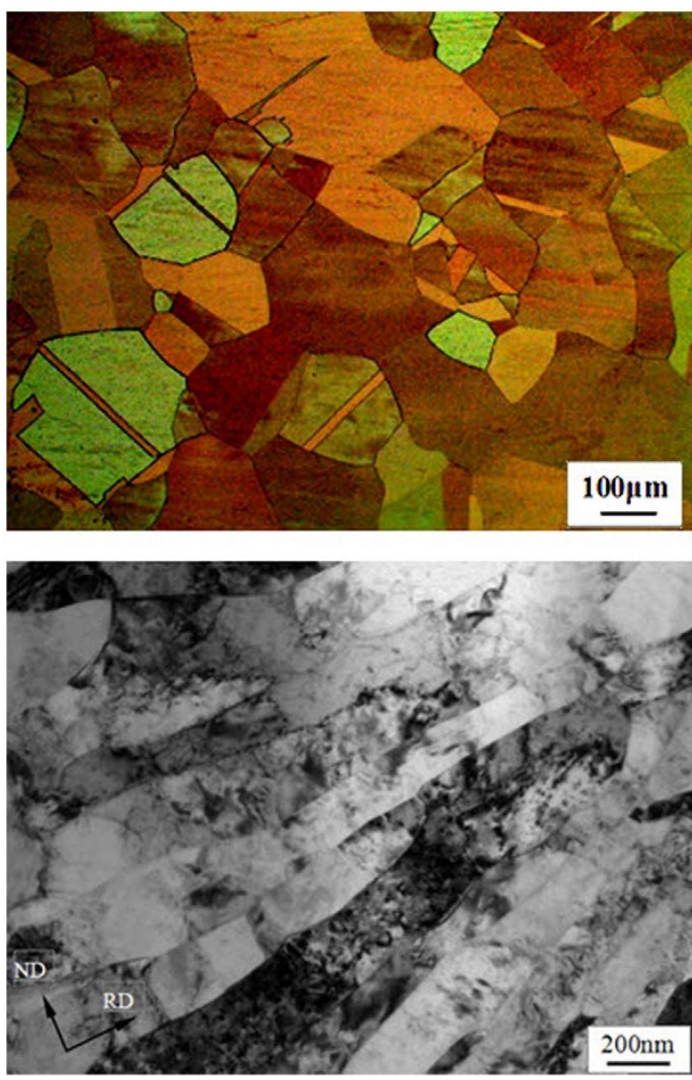

Figure $3 \mid$ Grains in the thickness direction of specimens at different ETR $\eta$. (a) OM: $\eta=0$; (b) TEM: $\eta=43,684 \%$.

when the ETR $\eta$ was in the range of $0-1,744 \%$ (i.e., specimen thickness in $8.3-0.45 \mathrm{~mm}$ ). The strain-hardening slowed down when $\eta$ was in the range of $1,744-8,200 \%$ (i.e., specimen thickness in 450-100 $\mu \mathrm{m}$ ), and the microhardness up to a peak of $137 \mathrm{HV} 0.05$. When $\eta>8,200 \%$ (i.e., specimen thickness below $100 \mu \mathrm{m}$ ), the strain-softening effect occurred. When $\eta=43,684 \%$ (i.e., specimen thickness of $19 \mu \mathrm{m}$ ), the microhardness was decreased to 84 HV0.05. The results indicate a wonderful phenomenon during rolling that microhardness was increased at first and then decreased later. Therefore, it is the strain softening to make the deformation easy and to avoid the strip breakage. It makes the forming process continue so as to obtain the ETR $\eta$ up to $43,684 \%$.

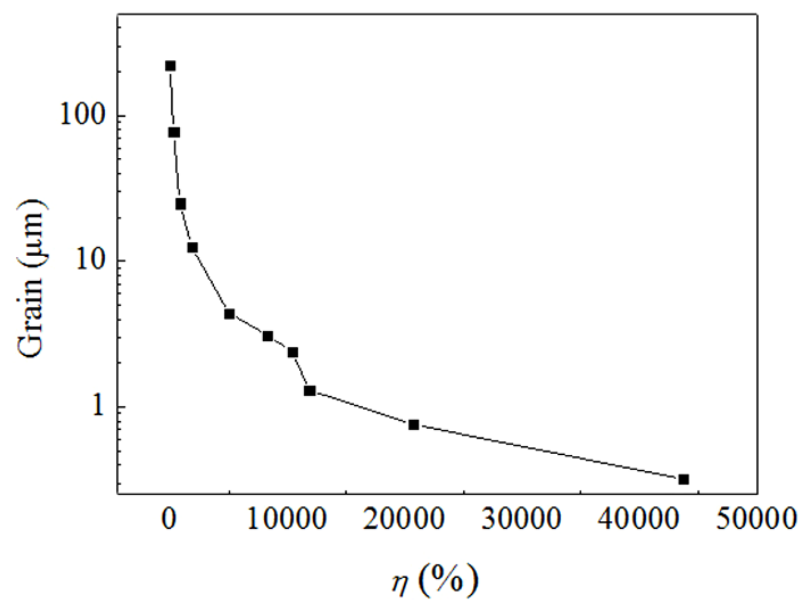

Figure $4 \mid$ Effect of ETR $\eta$ on the grain size in the thickness direction. 


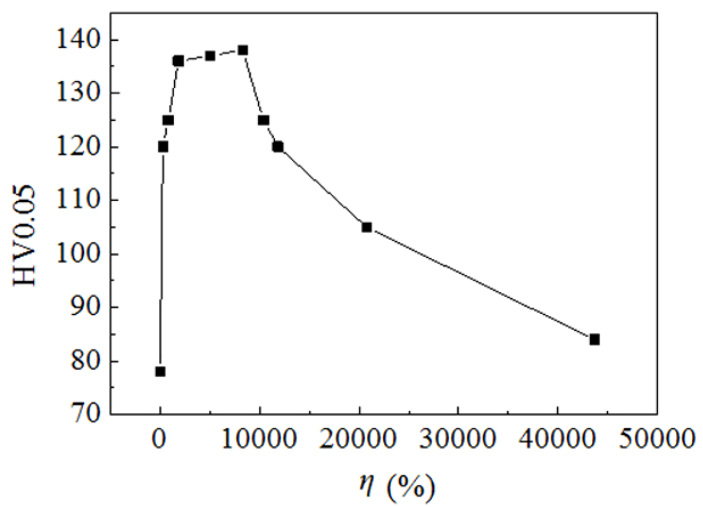

Figure 5 | Effect of ETR $\eta$ on the microhardness.

\section{Discussion}

The XRD patterns of an annealed $\mathrm{Cu}$ and the cold-rolled $\mathrm{Cu}$ with different ETR $\eta$ are shown in Figure 6, and the diffraction positions from the atomic planes are also presented. Compared to the annealed $\mathrm{Cu}$, some major reductions of the cold-rolled $\mathrm{Cu}$ with different ETR $\eta$ in the intensity of the (llll 1111$)$ and $\left(\begin{array}{lll}2 & 0 & 0\end{array}\right)$ peaks are observed (see Figure 6). The cold rolling process also causes the XRD peaks to broaden due to the refinement of grain size and the introduction of lattice strain. The integral breadth analysis has been given to calculate the grain size and lattice microstrain by Cauchy and Gaussian functions ${ }^{11}$ :

$$
\frac{(\delta 2 \theta)^{2}}{\operatorname{tg}^{2} \theta_{0}}=\frac{\lambda}{d}\left(\frac{\delta 2 \theta}{\tan \theta_{0} \sin \theta_{0}}\right)+25\left\langle\varepsilon^{2}\right\rangle
$$

where $\lambda$ is the wave length, $d$ is the average crystallite size, and $\varepsilon$ is the lattice strain, $\delta 2 \theta$ is the measured integral breadth, and $\theta_{0}$ the peak maximum position. For example, Figure 7 shows a least squares fit of the $(\delta 2 \theta)^{2} / \tan ^{2} \theta_{0}$ against $\delta 2 \theta /\left(\tan \theta_{0} \sin \theta_{0}\right)$ for all measured peaks of the cold-rolled $\mathrm{Cu}$ with $\eta=11,857 \%$. It can be seen that a good fit is obtained with 0.95 standard linear regression. The average crystallite size and the root mean square lattice strain were calculated to be $1.3 \mu \mathrm{m}$ and $0.0158 \%$.

For the materials subjected to severe plastic deformation, dislocation density $\rho$ can be represented in terms of grain size $d$ and microstrain $\left.<\varepsilon^{2}\right\rangle^{1 / 2}$ by the following equation ${ }^{12}$ :

$$
\rho=2 \sqrt{3}\left\langle\varepsilon^{2}\right\rangle^{1 / 2} /(d \times b)
$$

where $b$ is the Burgers vector and equals to $0.256 \mathrm{~nm}$ for a metal $\mathrm{Cu}$. The calculated $\rho$ of the cold-rolled $\mathrm{Cu}$ with $\eta=11,857 \%$ is $1.71 \times$ $10^{13} \mathrm{~m}^{-2}$. Similarly, the microstrain and dislocation density of the

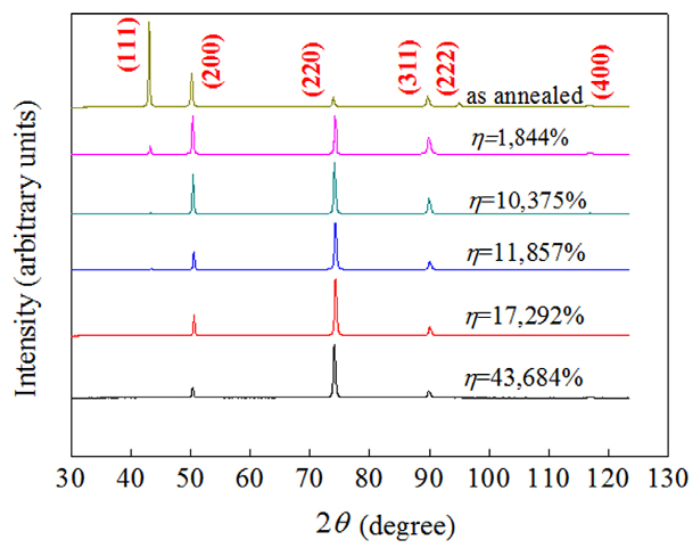

Figure 6 XRD patterns of the as-annealed and cold-rolled $\mathrm{Cu}$.

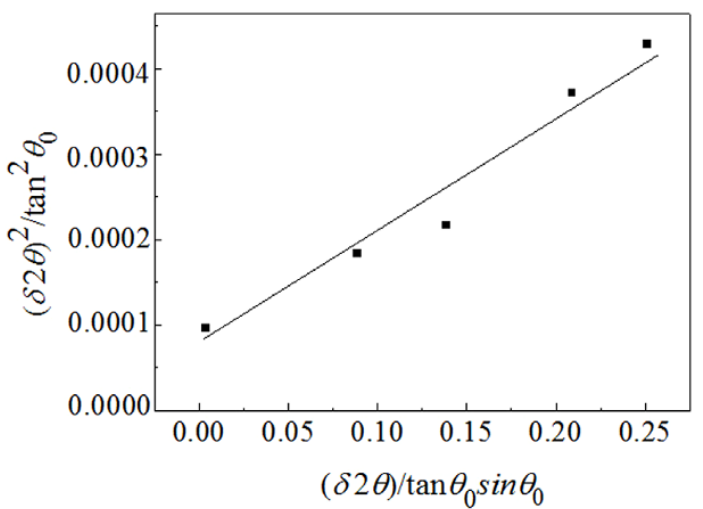

Figure $7 \mid$ Integral breadth analysis to calculate the grain size and the lattice strain from XRD data.

annealed $\mathrm{Cu}$ and the other cold-rolled $\mathrm{Cu}$ with different ETR $\eta$ are shown in Figure 8. It can be seen that the dislocation density is increased first When $\eta<8,200 \%$ and then deceased When $\eta>$ $8,200 \%$, indicating that the variation tendency of microstrain agrees very well with the hardness measurement results.

In order to deeply investigate the strain-softening mechanism in foil rolling, the TEM observation was carried out for the specimens with different ETR $\eta$, and the rolling plane was selected as the field of view. It was found that when $\eta=8,200 \%$ (the severest hardening), due to the heavy reduction by repeated rolling, the structure (Figure 9a) to consist of a cellular network with cell walls of high dislocation density, can be seen. This dislocation configuration indicates that the strain hardening is severe. When $\eta=43,684 \%$ (the most obvious strain softening), the cell structure turns to sub-grain (Figure 9b), the sub-grain boundaries are clear, and it is hard to find the dislocations.
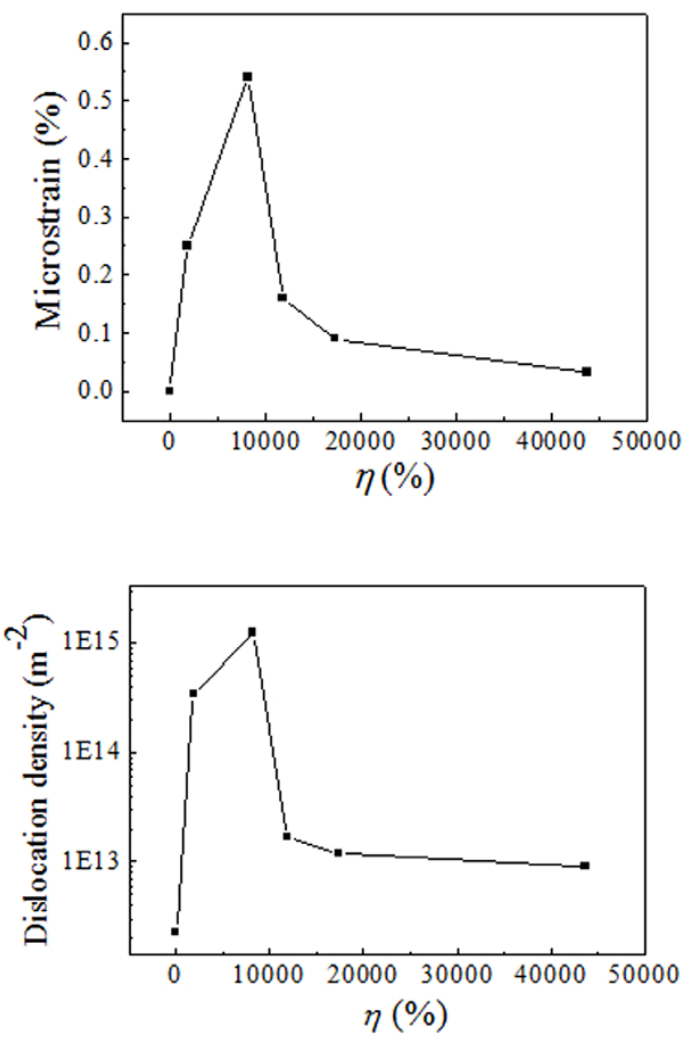

Figure $8 \mid$ XRD measurement results for the cold-rolled Cu with different ETR $\eta$. (a) Microstrain vs. ETR $\eta$; (b) dislocation density vs. ETR $\eta$. 

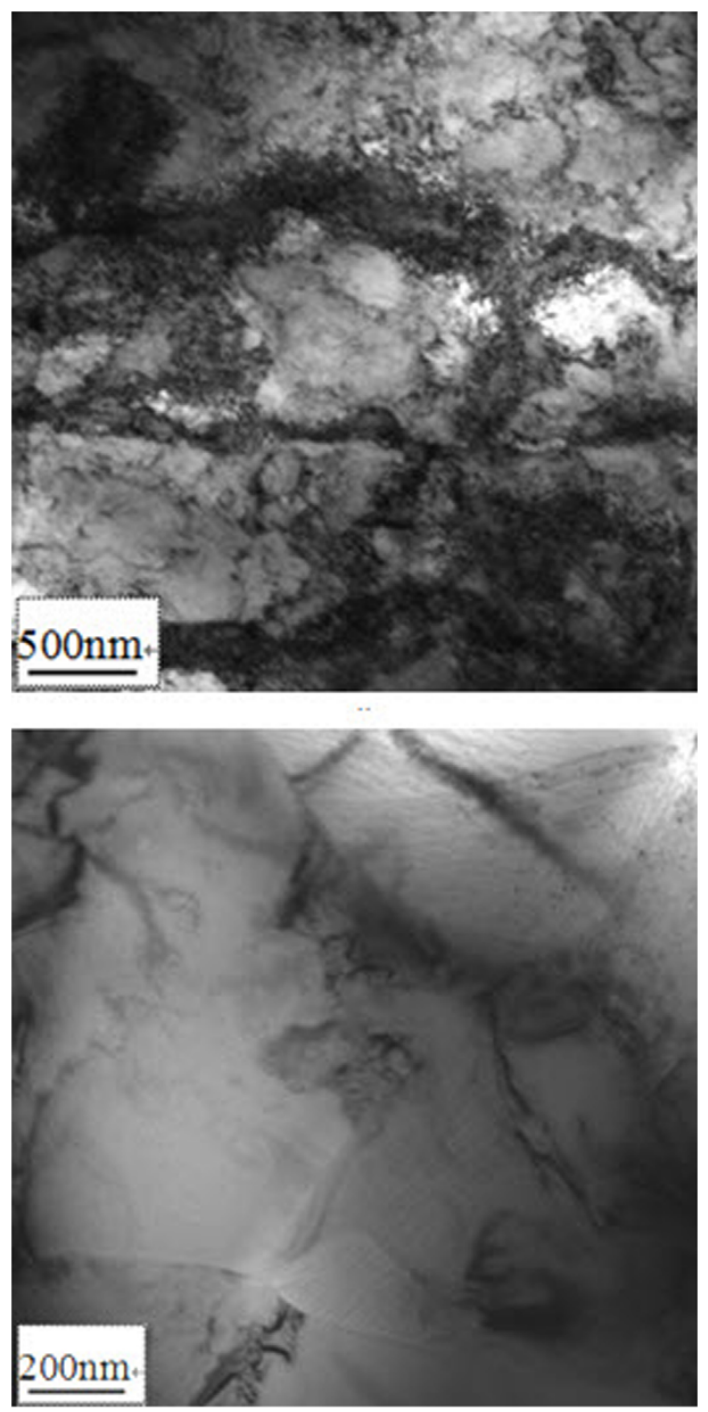

Figure 9 TEM images of the copper specimens with different ETR $\eta$. (a) $\eta=8,200 \%$; (b) $\eta=43,684 \%$.

In addition, TEM observation revealed the deformation twin (Figure 10) in local area. These results tell us that such extreme extensibility could be attributed to the combination action by dislocation evolution (is increased first, and then reduced) and twining. The strain softening is a performance corresponding to the extreme extensibility. It seems that there is a threshold value around $100 \mu \mathrm{m}$. Across this value, the strain-softening becomes more clearly with increasing the rolling reduction, and the following deformation is much easier than before. Finally the ETR $\eta$ is up to as high as $43,684 \%$. This interpretation agrees very well with the hardness measurement curve and TEM observation results to the dislocation and twining. Besides the copper, we found in our experiments that the steel and aluminum foil also revealed strain-softening phenomena during rolling at room temperature, when the specimen thickness is down to below $100 \mu \mathrm{m}$. According to the TEM observation shown in Figures 9 and 10, it is initially concluded that the strain softening is related with dislocation-configuration evolution and twining.

It has been indicated that as the foil thickness was decreased to dozens of micron level, the mechanical properties and damage behavior of the material could be changed ${ }^{13-18}$. Fundamental investigations, especially on the strain softening in thin metals forming, have been carried out and corresponding theories have been investigated $^{19-21}$. In order to explain the flow stress decreases with the decrease of specimen thickness, Kals T.A. et al. ${ }^{13}$ developed an

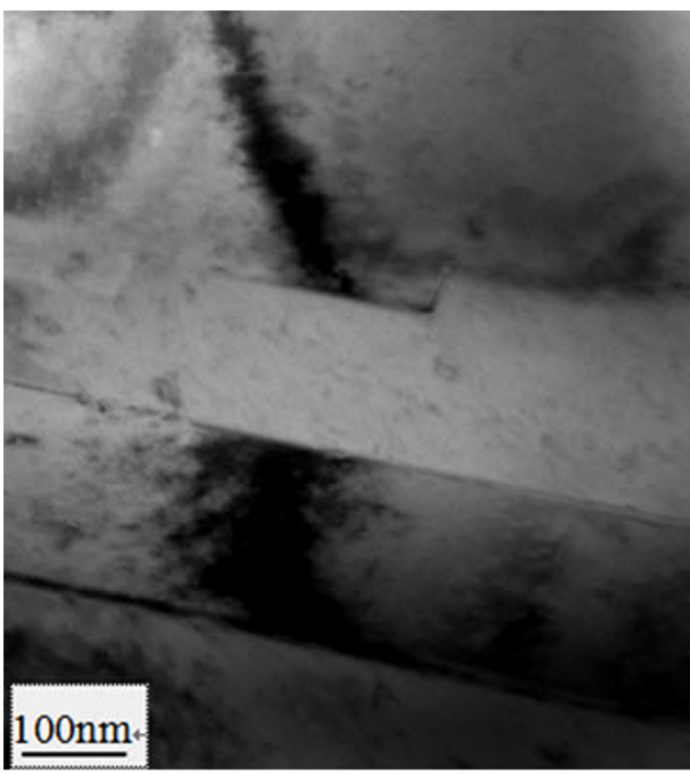

Figure 10 $\mid$ TEM observation of the ladder-type deformation twin. The twin thickness is about $180 \mathrm{~nm}$.

analytical model of the share of surface grains with CuNi18Zn20 and CuZn15, both in the soft recrystallized state. The model reveals that when the thickness of the specimen is reduced and the maintaining microstructure unchanged, it is found the ratio of the amount of the grains on specimen surface to that of the grains inner specimen increases. Meanwhile, because the constraint acting on the grains located at the specimen surface is weaker than that acting on the grains located within the specimen, the dislocations could slip out of the free surface of specimen instead of piling up at the surface, resulting in strain softening. Similarly, Lee H. J. et al. ${ }^{22}$ reported the results of microtensile tests and transmission electron microscopy analyses of micron-scale free-standing aluminum thin films. They found that in tensile testing for the $2 \mu \mathrm{m}$ thick $\mathrm{Al}$ films annealed at 250 and $350^{\circ} \mathrm{C}$ (less than two grains in the thickness), the dislocation loss to the surfaces makes grain interiors mechanically softer than near grain boundary regions.

Nevertheless, it must be mentioned that the dislocation loss mechanism on the strain softening of thin metals discussed above is correct under such a condition that the investigated thin metals must be recrystallized state to enable few coarse grains distribution in the thickness direction, which is main factor but usually neglected. That is an only way to make the dislocations within the specimen easily reach the free surfaces of specimen. However, with regard to the cold-rolled copper thin foil in our work, it is still hard to explain using the dislocation loss mechanism. Since the cold-rolled copper thin foil is composed of quite a few layers of squashed grains (without annealing) in the thickness direction because of repeated cold rolling (see Figure 3b), it is impossible for the dislocations from the grains located within the specimen to arrive the free surfaces of the grains located at the specimen surface due to the constraint of grain boundaries. Certainly, for such a thin foil composed of quite a few layers of squashed grains, the dislocation loss to the surface still takes place only at the grains of specimen surface. But the mechanical properties of the specimen mainly depend on a lot of squashed grains without dislocation loss within the specimen rather than surface grains. Therefore, the strain softening on the cold-rolled copper thin foil can not well explained by present theory.

In view of this, we search the reason from the viewpoint of dislocation. At the beginning of rolling deformation, it has enough room in the interior of coarse gains for the dislocations to slip, pile up and store, as shown in Figure 11(a). That means the dislocation density 


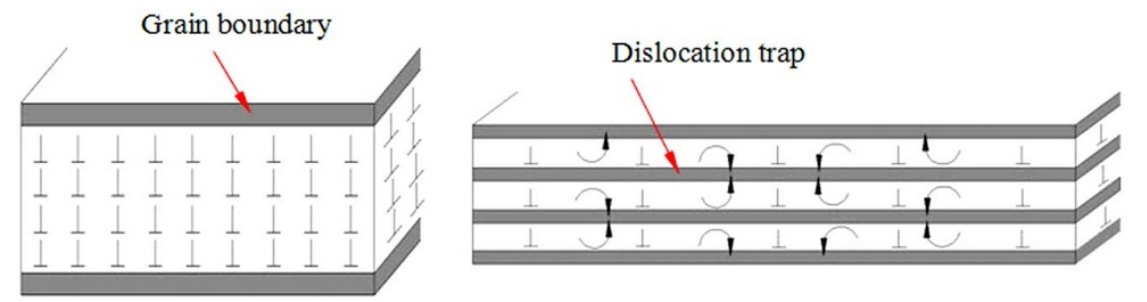

Figure 11 Schematic of (a) dislocation pileup within grain; (b) dislocation annihilation at the grain boundaries within specimen.

could increase from low to high level during cold rolling. Meanwhile, the hardness would increase, and then the strain hardening is observed. When the specimen thickness is down to a threshold below $100 \mu \mathrm{m}$, the grain layer is so thin (in the range of 2.4-0.24 $\mu \mathrm{m}$ ) as to be close or attain few micron dimension, so as to lead the dislocationstorage capacity of the grains to be poor. Additionally, the dislocation density within the grains in the thickness direction is saturated due to repeated rolling, and the ratio areas of grain boundary are increased as the ETR $\eta$ increases. These lead to the saturated dislocations could easily move to the nearest grain boundaries from the interior of a grain. It makes the unlike dislocations of the upper and lower adjacent grains meet together and annihilate each other. That means each layer of boundaries could be regarded as a dislocation trap (Figure 11b), which make the cumulative distortion energy release, and then strain softening become main tendency. Furthermore, the smaller the grain size in the thickness direction, dislocations lost at grain boundaries more obvious. The strain hardening or strain softening during the cold rolling of the copper thin foil depend on the competition between the dislocation multiplication and the dislocations lost to the grain boundaries within the specimen. As a result, we suggest that the strain softening of the thin metals does not always arise from the dislocation loss to the surfaces of specimen. On the contrary, the grain boundaries within the specimen suffered severely plastic deformation should also be regarded as dislocation traps.

Our success in obtaining such an extreme extensibility up to $43,684 \%$ at room temperature and without intermediate annealing in an industrial coarse-grained polycrystalline $\mathrm{Cu}$ specimen. Such an extreme extensibility is mainly attributed to the strain-softening effect, because the results show that with the increase of reduction, the specimen hardness increases up to a peak value $138 \mathrm{HV} 0.05$ when the $\eta=8,200 \%$ approximately, and then decreases to $78 \mathrm{HV} 0.05$ at final thickness $19 \mu \mathrm{m}$ instead. Furthermore, quantitative X-ray diffraction (XRD) measurements confirmed that the dislocation density increased in the initial stage $(\eta<8,200 \%)$ and then deceased $(\eta>$ $8,200 \%)$, indicating that the variation tendency of microstrain agrees very well with the hardness measurement results. Meanwhile, TEM observation also reveals that the dislocation pileup of specimen with $\eta=8,200 \%$ is severer compared to that of specimen with $\eta=$ $43,684 \%$. Although we know that it is possible to have other reasons for the strain softening, we still believe that the above dislocation annihilation mechanics play an important role in the strain softening and the extreme extensibility. In addition, we suggest that the strain softening of the thin metals does not always arise from the dislocation loss to the surfaces of specimen. On the contrary, the grain boundaries within the specimen suffered severely plastic deformation should also be regarded as dislocation traps.

\section{Methods}

Special rolling process. The rolling experiment was carried out on a four-high mill, which developed by ourselves and called as $3 \mathrm{M}$ mill (Micro-foil Multi-functions Mini mill, as shown in Fig. 1). It has the function of cross shear rolling, with free chosen speed ratio between up and down roll, and therefore the shearing action zone can be controlled by adjusting the speed ratio. The roll diameter is $50 \mathrm{~mm}$ for work rolls and $120 \mathrm{~mm}$ for backup rolls, and the roll barrel is $120 \mathrm{~mm}$. There are two coilers at the entry and exit of the mill, providing the front and back tension with high level upon the foil. By the $3 \mathrm{M}$-mill the compound forming conditions of compression, shearing and tension, can be obtained.
Specimen preparation for microhardness test and microstructure observation. The tested material is an industrial pure copper (99.9\%). It was annealed at $600^{\circ} \mathrm{C}$ for $1 \mathrm{~h}$ in a vacuum furnace to make the grains grow fully. The specimen was cut to $30 \times$ $36 \times 8.3 \mathrm{~mm}$ for rolling. The microhardness was measured with the load of $0.49 \mathrm{~N}$ for $15 \mathrm{~s}$. The test positions are on the rolled plane of specimens. Microstructures of the annealed specimen were investigated by optical microscopy after etching with a solution of $5 \mathrm{~g}$ of $\mathrm{FeCl}_{3}, 15 \mathrm{ml}$ of $\mathrm{HCl}$ and $85 \mathrm{ml}$ of $\mathrm{H}_{2} \mathrm{O}$ for $20-30 \mathrm{~s}$.

TEM experiment. Transmission electron microscopy (TEM) was carried out using a Tecnai G2 F20 microscope operating at $300 \mathrm{kV}$. The TEM specimens were prepared by mechanical grinding of the industrial pure $\mathrm{Cu}$ to a thickness of about $10 \mu \mathrm{m}$. Further thinning to a thickness of electron transparency was performed by a Gatan Dual Ion Milling System with an $\mathrm{Ar}+$ accelerating voltage of $4 \mathrm{kV}$ and liquid nitrogen for cooling the specimens.

Preparation of the TEM specimen using FIB. A FEI HELIOS NanoLab 600 Dualbeam system, including a focused ion beam (FIB) and a field emission scanning electron microscope (SEM), was used to prepare the thin-foil specimens from the $\mathrm{Cu}$ coils for further TEM observation. This system is equipped with an in-situ platinum deposition system, which provides the localized protection on the top surface against the ion beam damage. By this system, the electron beam was utilized to determine the zone of interest on the specimen surface. The FIB column forms an energetic beam of gallium ions, which scans over the specimen surface for imaging as well as ion milling. During the FIB milling, the accelerating voltage for rough cutting is $30 \mathrm{kV}$ and high ion beam currents is $5 \mathrm{nA}$, and the lower beam currents for polishing both sides of the thin membrane are $0.1 \mathrm{nA}$ to $1 \mathrm{nA}$.

XRD experiment. Quantitative X-ray diffraction (XRD) measurements were performed using a diffractometer (model D/MAX-RC) with a rotating copper anode $(\mathrm{CuK} \alpha)$ radiation (wavelength, $\mathrm{k}=0.15405 \mathrm{~nm}$ ). The $\mathrm{X}$-ray beam size is about $0.185 \mathrm{~mm}$ on the specimen, and the scattered radiation is registered by four different position-sensitive imaging detectors located at 300 to $600 \mathrm{~mm}$ from the base material. The scattered radiation covered about $2 \theta=30$ to 140 degrees of the angular range of diffractions.

1. Isaac Samuel, E. \& Choudhary, B. K. Tensile work hardening behaviour of P91 steel. Mater. Sci. Eng. A 528, 7827-7830 (2011).

2. Renard, K. \& Jacques, P. J. On the relationship between work hardening and twinning rate in TWIP steels. Mater. Sci. Eng. A 542, 8-14 (2011).

3. Roumina, R. \& Sinclair, C. W. The work hardening rate of an aged and recovered Al-Mg-Sc alloy. Metall Mat Trans Phys Metall Mat Sci 42, 473-487 (2011).

4. Li, F. Z., Liu, Z. J., Jin, Q. \& Yu, Z. M. Work-softening mechanism of pure aluminums and Al-Fe alloys. Zhongguo Youse Jinshu Xuebao 7, 98-102 (1997).

5. Jin, Q., Liu, Z. J., Yu, Z. M. \& Li, F. Z. Studies on working softening of pure aluminium. Journal Harbin Univ. Sci. \& Tech. 17, 28-31 (1993).

6. Lu, L., Sui, M. L. \& Lu, K. Superplastic extensibility of nanocrystalline copper at room temperature. Science 287, 1463-1466 (2000).

7. Guo, J. Y., Wang, K. \& Lu, L. Tensile properties of $\mathrm{Cu}$ with deformation twins induced by SMAT. J. Mater. Sci. Technol. 22, 789-792 (2006).

8. Lu, Y. F. et al. Interface and size effects in metal nano-composites. Zhongguo Youse Jinshu Xuebao 22, 1650-1658 (2012).

9. Lu, K. The future of metals. Science 328, 319-320 (2010).

10. Huang, T. et al. Effect of speed ratio on textures transformation in cross shear rolled high purity aluminum foils. Zhongguo Youse Jinshu Xuebao 16, 110-115 (2006).

11. Youssef, K. M. et al. Nanocrystalline Al-Mg alloy with ultrahigh strength and good ductility. Scripta Mater. 54, 251-256 (2006).

12. Zhao, Y. H. et al. Microstructures and mechanical properties of ultrafine grained $7075 \mathrm{Al}$ alloy processed by ECAP and their evolutions during annealing. Acta Mater. 52, 4589-4599 (2004).

13. Kals, T. A. \& Eckstein, R. Miniaturization in sheet metal working. J. Mater. Process. Technol. 103, 95-101 (2000).

14. Michel, J. F. \& Picart, P. Size effects on the constitutive behaviour for brass in sheet metal forming. J. Mater. Process. Technol. 141, 439-446 (2003).

15. Raulea, L. V. et al. Size effects in the processing of thin metal sheets. J. Mater. Process. Technol. 115, 44-48 (2001). 
16. Trelewicz, J. R. \& Schuh, C. A. The Hall-Petch breakdown in nanocrystalline metals: A crossover to glass-like deformation. Acta Mater. 55, 5948-5958 (2007).

17. Aifantis, K. E. \& Konstantinidis, A. A. Hall-Petch revisited at the nanoscale. Mater Sci Eng B Solid State Adv Technol 163, 139-144 (2009).

18. Lee, H. J., Zhang, P. \& Bravman, J. C. Study on the strength and elongation of freestanding $\mathrm{Al}$ beams for microelectromechanical systems applications. Appl. Phys. Lett. 84, 915-917 (2004).

19. Miyazaki, S., Fujita, H. \& Hiraoka, H. Effect of specimen size on the flow stress of rod specimens of polycrystalline cu-al alloy. Scr Metall 13, 447-449 (1979).

20. Fu, M. W. \& Chan, W. L. Geometry and grain size effects on the fracture behavior of sheet metal in micro-scale plastic deformation. Mater. Des. 32, 4738-4746 (2011)

21. Kusnierz, J., Kurowski, M. \& Baliga, W. Strain softening effects in microstructure of twisted pre-deformed copper rods. Mater. Chem. Phys. 81, 548-551 (2003).

22. Lee, H. J., Zhang, P. \& Bravman, J. C. Tensile failure by grain thinning in micromachined aluminum thin films. J. Appl. Phys. 93, 1143-1151 (2003).

\section{Acknowledgments}

The authors thank the "333 Project" Training Foundation of Jiangsu Province of China (grant BRA2011037), the "Six Talent Peaks" Project of Jiangsu Province of China (grant 2012-ZBZZ-007), the Natural Science Foundation of Jiangsu Province of China (No.
BK20131123), and the National Natural Science Foundation of China No. 51374069. The authors also want to thank Mr. Meng Song in Northeastern University, Shenyang, China for his help in XRD and TEM observation.

\section{Author contributions}

X.H.L. and Q.B.Y. designed and directed the overall study. Rolling experiments were carried out by Q.B.Y. and D.L.T. Q.B.Y. wrote the manuscript and discussed the results and analyzed the data with X.H.L. Q.B.Y. was responsible for OM and TEM.

\section{Additional information}

Supplementary information accompanies this paper at http://www.nature.com/ scientificreports

Competing financial interests: The authors declare no competing financial interests.

How to cite this article: Yu, Q.B., Liu, X.H. \& Tang, D.L. Extreme Extensibility of Copper Foil under Compound Forming Conditions. Sci. Rep. 3, 3556; DOI:10.1038/srep03556 (2013)

(c) (i) $(-)$ This work is licensed under a Creative Commons AttributionNonCommercial-NoDerivs 3.0 Unported license. To view a copy of this license, visit http://creativecommons.org/licenses/by-nc-nd/3.0 